\title{
Implications for psychedelic-assisted psychotherapy: functional magnetic resonance imaging study with psilocybin
}

R. L. Carhart-Harris, R. Leech, T. M. Williams, D. Erritzoe, N. Abbasi, T. Bargiotas,

P. Hobden, D. J. Sharp, J. Evans, A. Feilding, R. G. Wise and D. J. Nutt

\section{Background}

Psilocybin is a classic psychedelic drug that has a history of use in psychotherapy. One of the rationales for its use was that it aids emotional insight by lowering psychological defences.

\section{Aims \\ To test the hypothesis that psilocybin facilitates access to personal memories and emotions by comparing subjective and neural responses to positive autobiographical memories under psilocybin and placebo.}

\section{Method}

Ten healthy participants received two functional magnetic resonance imaging scans (2mg intravenous psilocybin $v$. intravenous saline), separated by approximately 7 days, during which they viewed two different sets of 15 positive autobiographical memory cues. Participants viewed each cue for $6 \mathrm{~s}$ and then closed their eyes for $16 \mathrm{~s}$ and imagined re-experiencing the event. Activations during this recollection period were compared with an equivalent period of eyes-closed rest. We split the recollection period into an early phase (first $8 \mathrm{~s}$ ) and a late phase (last $8 \mathrm{~s}$ ) for analysis.

\section{Results}

Robust activations to the memories were seen in limbic and striatal regions in the early phase and the medial prefrontal cortex in the late phase in both conditions $(P<0.001$, whole brain cluster correction), but there were additional visual and other sensory cortical activations in the late phase under psilocybin that were absent under placebo. Ratings of memory vividness and visual imagery were significantly higher after psilocybin $(P<0.05)$ and there was a significant positive correlation between vividness and subjective wellbeing at follow-up $(P<0.01)$.

\section{Conclusions}

Evidence that psilocybin enhances autobiographical recollection implies that it may be useful in psychotherapy either as a tool to facilitate the recall of salient memories or to reverse negative cognitive biases.

\section{Declaration of interest}

None.
Psilocybin is a classic psychedelic ('mind-manifesting') drug, pharmacologically related to the prototypical psychedelic, lysergic acid diethylamide. Psychedelic drugs were used extensively in psychotherapy in the 1950s to lower psychological defences and facilitate emotional insight. ${ }^{1}$ In cognitive terms, the 'lowering of defences' may be thought of as a decrease in top-down emotional control. There are several reports in this literature of spontaneous autobiographical recollections or 'relivings' under psychedelics ${ }^{2}-$ similar in some respects to the dream-like sequences seen on stimulation of the medial temporal lobes ${ }^{3}$ or to the flashback phenomena seen in post-traumatic stress disorder (PTSD). ${ }^{4}$ In a previous psilocybin functional magnetic resonance imaging (fMRI) study by our group, ${ }^{5}$ one individual reported a striking reliving under the drug, further motivating us to test this phenomenon in a controlled manner. We also observed large decreases in resting state activity in the medial prefrontal cortex after psilocybin. ${ }^{5}$ The medial prefrontal cortex is known to exert top-down inhibitory control over limbic activity, ${ }^{6}$ so a psilocybininduced deactivation of the medial prefrontal cortex - leading to a disinhibition of limbic activity - may explain the occurrence of spontaneous recollections in the psychedelic state.

Thus, the present study sought to test the hypothesis that psychedelic drugs facilitate autobiographical recollection, using psilocybin and a blocked fMRI paradigm involving personal memory cues. Spontaneous relivings under psychedelics are often explicitly linked to past traumata; ${ }^{2}$ however, we used only positive memory cues in order to minimise the risk of adverse reactions.
We predicted that psilocybin would augment subjective and neural responses to personal memories. Based on previous studies implicating medial temporal and visual association regions in vivid autobiographical recollections, ${ }^{7-9}$ we predicted that psilocybin would increase activations in these specific regions.

\section{Method}

\section{Design}

This was a placebo-controlled cross-over study approved by a local National Health Service research ethics committee and research and development body, and conducted in accordance with good clinical practice guidelines. A Home Office licence was obtained for storage and handling of a schedule 1 drug. The University of Bristol sponsored this research.

\section{Participants}

Fifteen healthy individuals were recruited, all via word of mouth. However, only 13 completed the behavioural component of the fMRI study because of presentation software failure for 2 individuals after administration of psilocybin, and three further data-sets were removed because of scanner artefacts, rendering the functional data unusable. Of the ten remaining participants, nine were male, all were physically and mentally healthy, with a mean age of 31 years (s.d. $=7.5)$, and all had used psilocybin before (average 16.4 uses, s.d. $=27.2$ ), but not within 6 weeks of the 
study. Participants attended a screening visit during which their mental and physical health was assessed. This included a brief psychiatric interview, routine blood analysis, electrocardiogram, blood pressure and heart rate, and a neurological examination.

\section{Derivation of autobiographical memory cues}

After enrolment, participants were given at least 2 weeks to provide 30 or more personal memories for use in the fMRI behavioural paradigm. They were instructed that these should refer to specific life events, and not general periods of time, and that they should be especially positive and emotionally salient. To avoid embarrassment, individuals were advised to encrypt especially personal memories so that they could only be understood by themselves. Cues were abridged to one sentence (e.g. 'Remember being at the altar getting married'). To control for recency effects, participants were asked to estimate the approximate age they were at the time of the event, and to rate the emotionally potency of the memory on a scale of $0-3$, with 3 being 'especially potent' (i.e. capable of eliciting a strong emotionally response and/or vivid recollection). The 30 most potent memories were split into 2 groups of 15 memories and balanced for recency and potency. Each group was randomly assigned to either the psilocybin or placebo condition.

\section{Scanning procedure and drug administration}

Participants attended two scanning sessions, separated by at least 7 days. Psilocybin ( $2 \mathrm{mg}$ in saline) was received on one day, and placebo (saline only) on the other, in a balanced order across participants. Solutions $(10 \mathrm{ml})$ were administered during scanning, as $60 \mathrm{~s}$ intravenous infusions.

Participants were cannulated and screened for magnetic resonance compatibility. Prior to functional scanning, we obtained an initial three-dimensional SPGR structural scan in an axial orientation, with field of view $256 \times 256 \times 192 \mathrm{~mm}$ and matrix $256 \times 256 \times 192 \mathrm{~mm}$ to yield $1 \mathrm{~mm}$ isotropic voxel resolution (repetition time (TR)/echo time (TE) 7.9/3.0 ms; inversion time $450 \mathrm{~ms}$; flip angle $20^{\circ}$ ). All scanning was performed on $3 \mathrm{~T} \mathrm{GE}$ $\mathrm{HD} \times$ MR system.

Blood oxygen level-dependent (BOLD)-weighted fMRI data were acquired using a gradient echo-planar imaging sequence, TR/TE $3000 / 35 \mathrm{~ms}$, field of view $192 \mathrm{~mm}, 64 \times 64$ acquisition matrix, parallel acceleration factor 2, and flip angle $90^{\circ}$. Fifty-three oblique-axial slices were acquired in an interleaved fashion, each $3 \mathrm{~mm}$ thick with zero slice gap $(3 \times 3 \times 3 \mathrm{~mm}$ voxels $)$. An initial task-free, eyes-closed, resting state scan took place for $12 \mathrm{~min}$ in total. The data from these scans will be presented in a separate publication. Sixty-second manual infusions of either psilocybin or placebo began $6 \mathrm{~min}$ after the start of the resting-state scan and the behavioural scan began $7.5 \mathrm{~min}$ after the start of the infusion. The subjective effects of intravenous psilocybin $(2 \mathrm{mg})$ peak a few minutes after infusion and persist for approximately 25-30 min (online Fig. DS1 and Carhart-Harris et $a l^{10}$ ); thus, the subjective effects of psilocybin were robust throughout the behavioural scan. The total duration of the behavioural scan was $18.5 \mathrm{~min}$ (370 whole brain volumes).

\section{Functional paradigm}

Fifteen memory cues were presented in a blocked paradigm, interleaved with rest and an auditory attention task. The attention task was designed to investigate hypotheses unrelated to autobiographical memory and so the results are not included here. For the memory task, participants were instructed to close their eyes and to open them when cued by a loud auditory tone played through stereo headphones. Individuals were instructed to allow their memories to come back to them during a period of eyes-closed recollection, and not to resist any associated thoughts. Visual stimuli were projected onto a screen behind them which was viewed through a mirror attached to the head coil.

Participants performed the autobiographical memory condition with their eyes closed in order to aid recollection; eyes were also closed in the rest blocks to provide an unbiased baseline state, matched for sensory input. In the rest trials, individuals were first presented with an auditory tone and the visual instruction 'relax', which remained on the screen for $6 \mathrm{~s}$ followed by 'close your eyes'. Participants closed their eyes for $16 \mathrm{~s}$ before hearing an auditory tone which signalled for them to open their eyes. They were then presented with their first memory cue (e.g. 'Remember playing Oliver in the school play'), which was shown for $6 \mathrm{~s}$ before the instruction 'close your eyes'. Individuals were given $16 \mathrm{~s}$ to recollect the event before hearing an auditory tone signalling for them to open their eyes. They were then presented with the instruction 'count the beeps', which was shown for $6 \mathrm{~s}$, followed by 'close your eyes' and the onset of the attention task. This task consisted of a sequence of auditory tones of different pitch playing in either ear in a random order. Each participant had to decide whether more tones occurred in their left or right ear and gave their response via button press at the end of the $16 \mathrm{~s}$. There was always one more tone in either the left or the right ear. The fixed sequence of rest-memory-attention was repeated 15 times in total. The rationale for the fixed order was to minimise carry-over effects from the memory trials to the rest trials by using the attention paradigm to distract participants from sustained recollection of the memories.

\section{Subjective ratings}

Memory ratings were given after the participants exited the scanner. Each memory was rated for vividness ('How vivid was the memory, if 10 is extremely vivid - i.e. as if you are there experiencing it again?'), emotional intensity ('How strong was the emotion you felt, with 10 being extremely intense?'), valence ('How positive was the memory, if 10 is extremely positive, 5 is neither positive or negative, and 0 is extremely negative?') and visual imagery ('How visual was the memory, if 10 is extremely visual, as if you can see it again?'). Ratings were always given on a $0-10$ scale. Descriptive statistics were derived and $t$-tests were performed for between-condition comparisons. Participants were followed up 2 weeks after their scans and asked to rate any changes in their subjective well-being or life satisfaction on a scale of -3 (decreased very much) to +3 (increased very much), with 0 as 'no change'.

\section{Imaging analysis}

All imaging analysis was carried out using FSL (www.fmrib.ox. ac.uk/fsl). In the first-level analysis, a general linear model with four regressors was fitted to each participant. There was a regressor for the $16 \mathrm{~s}$ of eyes-closed attention to beeps and a regressor of no interest for the periods when the task instructions were presented and for when task responses were required. Previous imaging work on autobiographical recollection has found that different neural systems are engaged immediately after the memory cue compared with a few seconds later. ${ }^{9}$ Therefore, two regressors were used for the $16 \mathrm{~s}$ memory recollection period - one for the first $8 \mathrm{~s}$ after the memory cue (early phase) and one for the remaining $8-16 \mathrm{~s}$ (late phase). The two primary contrasts of interest were the early memory period $v$. rest, and the late memory period $v$. rest. The regressors were convolved using a 
double gamma response function, a temporal derivative was added and temporal filtering applied. Data were high-pass filtered with a cut off of $100 \mathrm{~s}$. The functional data were spatially smoothed $(6 \mathrm{~mm})$ and registered to each participants' $T_{1}$-weighted high-resolution $(1 \times 1 \times 1 \mathrm{~mm})$ structural scan which was itself registered to the Montreal Neurological Institute standard brain $(1 \times 1 \times 1 \mathrm{~mm})$ using non-linear registration.

The results of fitting this model at the first level were combined in a higher-level within-participants analysis to compare brain activation under psilocybin with activation under placebo. A mixed-effects analysis was performed to produce group statistics. Statistical parametric maps were cluster thresholded and a whole-brain cluster significance threshold of $P<0.05$ was used. To determine the mean activation maps for recollection $v$. rest under psilocybin and placebo, group means were derived for each condition. FSL fMRI Expert Analysis Tool (FEAT) query was used to compute BOLD per cent signal change for specific contrasts. Pearson's correlations were used to compare regional BOLD changes with subjective ratings - and specific variables were chosen on a hypothesis-driven basis. Given that recollection and emotion is typically thought to involve medial temporal lobe and limbic structures, anatomical regions of interest were created for bilateral parahippocampal gyrus and the amygdala based on the Harvard-Oxford probabilistic atlas.

\section{Results}

\section{Subjective ratings}

Memories were rated as more vivid, visual, emotional and positive under psilocybin than placebo (Fig. 1). Individually, only ratings of vivid ( $t$-test, $P=0.049$, two-tailed) and visual ( $t$-test, $P=0.041$, two-tailed) were significantly different, but if the four parameters (which were highly correlated) are grouped, ratings were significantly higher under psilocybin than placebo ( $t$-test, $P=0.0003$, two-tailed).

\section{fMRI results}

\section{Activations to memories}

Robust activations were observed during cued autobiographical recollection $v$. rest under both placebo and psilocybin, even with a stringent threshold of $Z=2$ (whole brain cluster corrected, $P<0.001$; Fig. 2). The contrasts were split up into an early phase recollection period versus rest (orange clusters) and a late phase

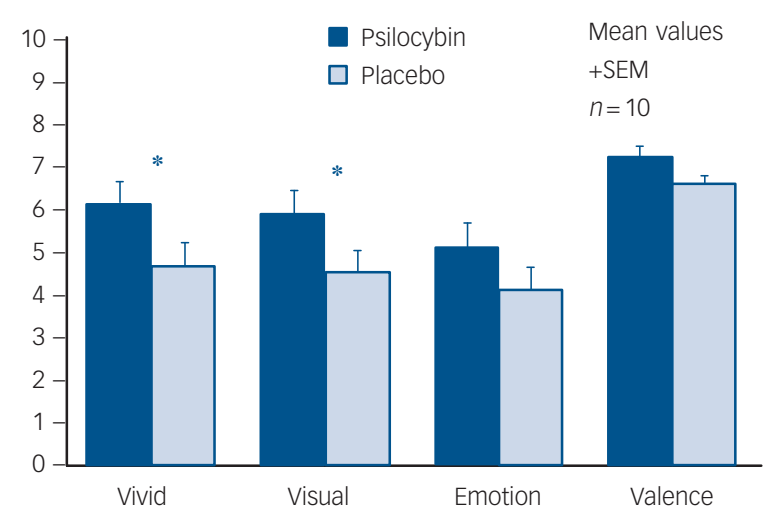

Fig. 1 Memory ratings after psilocybin and placebo.

Ratings were significantly higher after psilocybin than placebo for the items 'How vivid was the memory?' and 'How visual was the memory?' (two-tailed $t$-test, $\left.{ }^{*} P<0.05\right)$ and when all four were grouped $(P=0.0003)$. recollection period $v$. rest (dark red clusters); these were the first and last $8 \mathrm{~s}$ of the $16 \mathrm{~s}$ recollection period. The early activations were generally more subcortical - and were especially significant in limbic/medial temporal lobe (e.g. amygdala and hippocampus) and striatal regions (e.g. the putamen and nucleus accumbens), as well as the mid-cingulate cortex, pre-sensorimotor area and precuneus. Late activations were seen in limbic (e.g. the amygdala) and paralimbic regions (e.g. the subgenual cingulate cortex and pre-sensorimotor area) and the temporal pole, but there were also large activations in the medial prefrontal cortex and frontal pole that were notably absent in the early recollection period.

\section{Correlations with subjective ratings}

Combining the data from each individuals' two scans, there was a significant positive correlation between 'emotion' scores and late phase bilateral parahippocampal activations (Fig. 3, left; $r=0.4$, $P=0.04$, one-tailed). There was also a trend-level positive correlation between 'vividness' scores and late phase anterior bilateral parahippocampal activations ( $r=0.35, P=0.07$, one-tailed).

\section{Effect of psilocybin on activations to memories}

There were no significant differences between early phase activations to memories under psilocybin v. placebo. However, there were significantly greater late phase activations under psilocybin than placebo (Fig. 4). These were found in three large clusters, a left occipital pole and visual association region cluster (Fig. 3) and left and right hemisphere clusters that included the mid-insula and primary and secondary auditory cortex, the temporal pole, primary and secondary somatosensory cortex and superior parietal regions (e.g. the superior parietal lobule and supramarginal gyrus). Contrary to prior hypotheses, there were no between-condition differences in medial temporal lobe activations.

Since secondary sensory and high-level attention areas are typically deactivated during periods of introspection, ${ }^{11}$ we considered the possibility that the putative activations in these regions under psilocybin were in fact deactivations under placebo. To assess this, we looked at the first-level results for both conditions and calculated the mean percentage BOLD signal change to memories $v$. rest in the three above-mentioned clusters (Fig. 4). One cluster was in the left occipital pole and visual association regions (red in Fig. 4), one was in the left primary and secondary auditory cortex and left somatosensory and superior parietal areas (blue in Fig. 4), and one was in the right primary and secondary auditory cortex and right somatosensory and superior parietal areas (green in Fig. 4). After calculating the mean signal changes in each cluster, large deactivations were evident in the left and right hemisphere clusters under placebo (blue and green, Fig. 4), but true activations under psilocybin were found for all of the clusters - especially the visual one (red, Fig. 4), confirming that visual and other sensory regions are activated by memory cues under psilocybin.

\section{Follow-up ratings of well-being}

When data from the complete sample were analysed ( $n=15$, mean age 30.5 years (s.d. = 7.7), 2 females), reports of well-being 2 weeks after each scan were significantly higher after psilocybin than placebo $(P=0.03$, two-tailed). To test the hypothesis that participants with the most pronounced subjective responses to positive memories have the largest increases in well-being after psilocybin, we plotted scores of memory vividness against scores of increased well-being post-psilocybin for the 13 participants that completed the memory task in both conditions (Fig. 5). One outlier was removed owing to an explicit non-drug-related event 


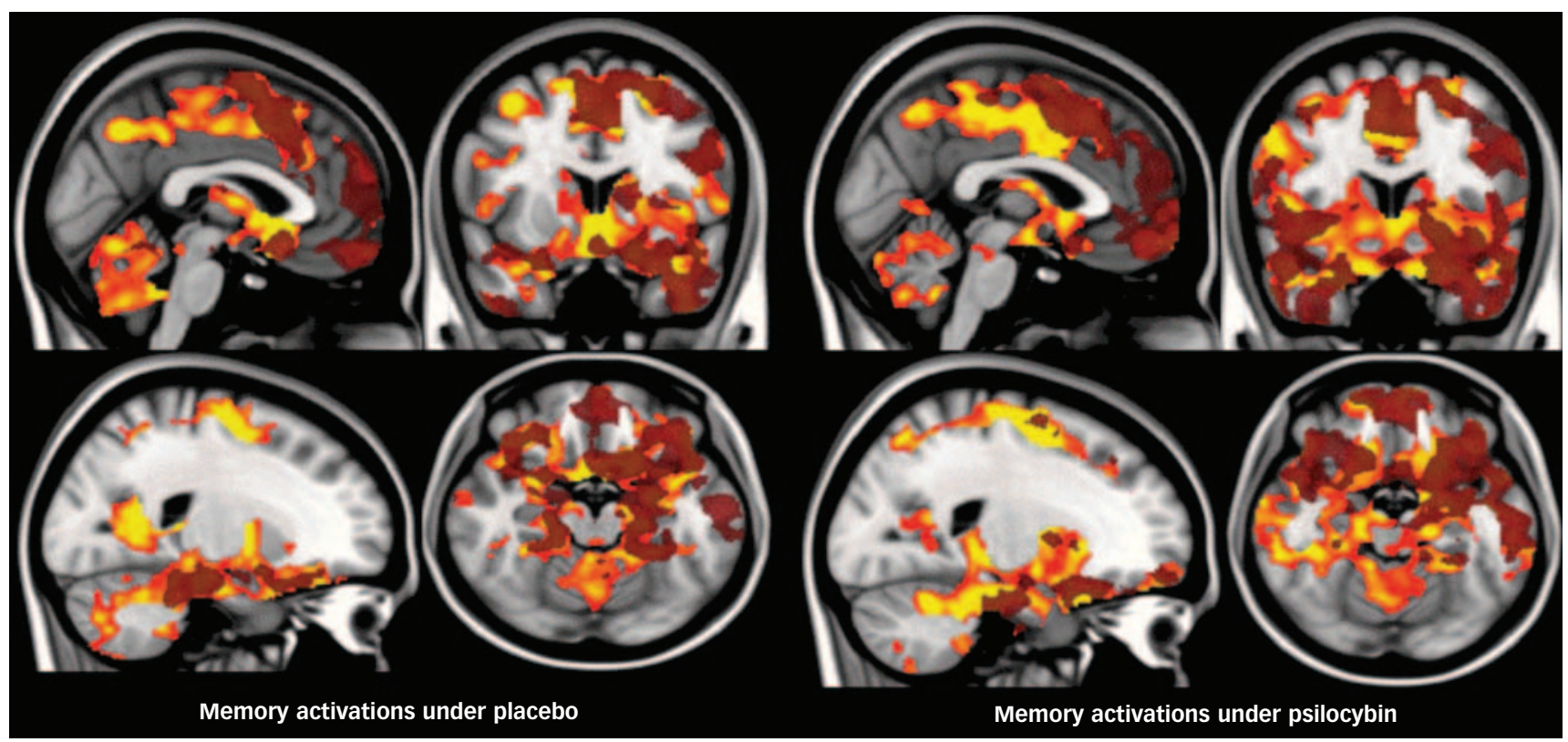

Fig. 2 Activations during autobiographical recollection v. rest under placebo and psilocybin.

Early phase activations are shown in orange and late phase activations in translucent dark red. Cluster threshold $Z=2$, whole brain corrected $P<0.001$. The left hemisphere is shown on the right.

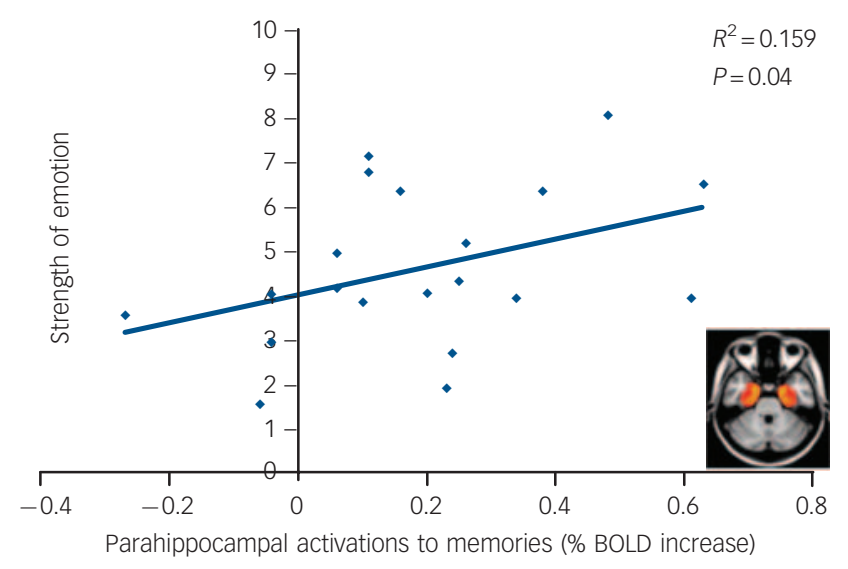

Fig. 3 Late phase parahippocampal (right) activations correlated positively with how emotional the memories were.

BOLD, blood oxygen level-dependent. Pearson's correlation, $P<0.05$, one-tailed.

causing him to report an anomalous decrease in well-being postpsilocybin. A significant positive correlation was found between vividness ratings and improved well-being post-psilocybin ( $r=0.72, P=0.004$, one-tailed).

\section{Discussion}

This study sought to test the hypothesis that psychedelics facilitate the neural processes underlying autobiographical recollection using fMRI and the classic psychedelic psilocybin. Robust activations to autobiographical memory cues were found after both placebo and psilocybin, but greater late phase sensory activations and more intense subjective effects were seen after psilocybin. Greater activations were observed in the bilateral auditory cortex, somatosensory cortex, superior parietal cortex, left visual association regions and the occipital pole after psilocybin, and

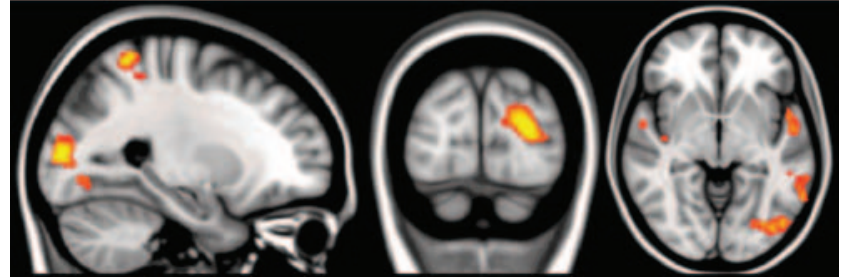

Fig. 4 Greater late phase activations during autobiographical recollection under psilocybin than placebo.

Cluster threshold $Z=2$, whole brain corrected $P<0.05$. The left hemisphere is shown on the right.

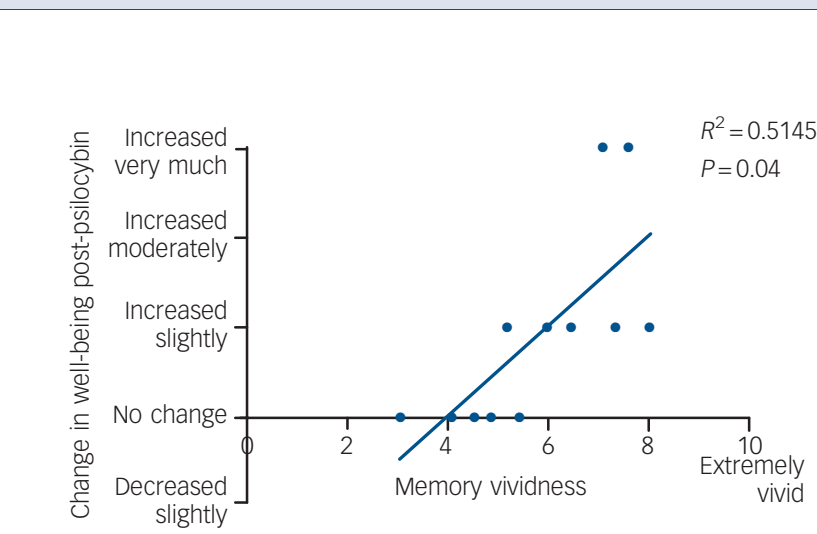

Fig. 5 Significant positive correlation between ratings of increased well-being post-psilocybin and ratings of memory vividness.

$r=0.72, P=0.004$, one-tailed

post-hoc tests confirmed that visual and other sensory regions were uniquely activated under psilocybin (Fig. 6). This switch in sensory function from a pattern of deactivation under placebo to activation under psilocybin is important and may explain why memories can be felt as especially vivid or 'real' under psychedelics). ${ }^{2,12,13}$ 

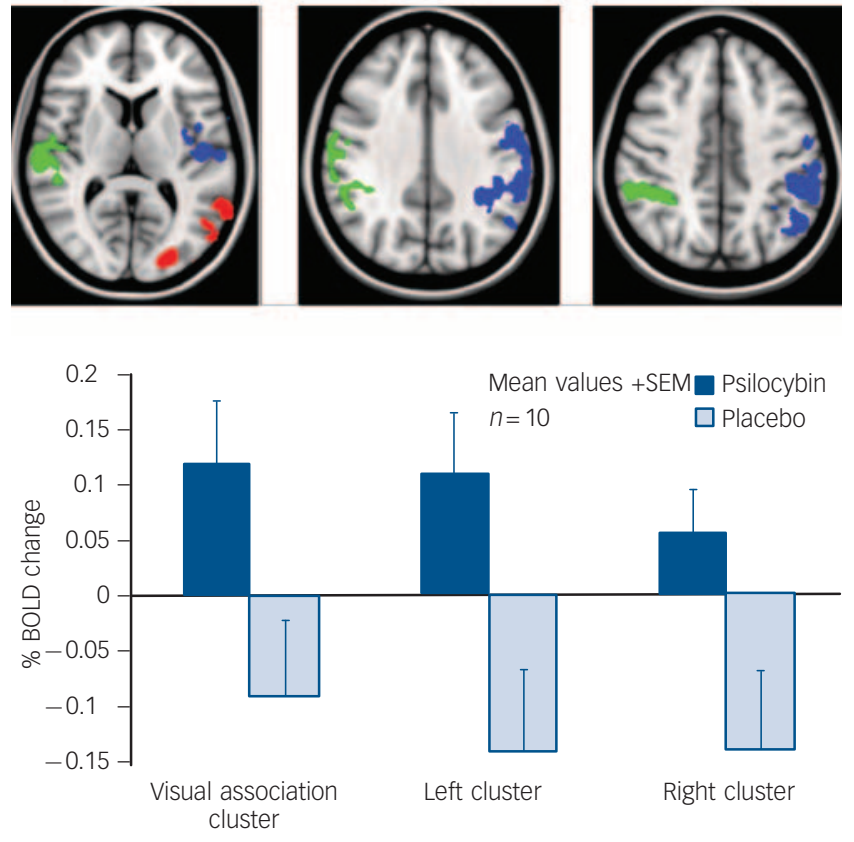

Fig. 6 Mean per cent blood oxygen level-dependent (BOLD) signal changes to memories $v$. rest under psilocybin (blue) and placebo (grey).

The left hemisphere is shown on the right.

\section{Relevance to previous studies}

Memory activations in both conditions were similar to those found in previous studies. Medial temporal activations are a relatively reliable finding in autobiographical recollection paradigms ${ }^{6}$ and have also been shown to correlate with emotional intensity $^{7}$ (as shown in Fig. 3). Moreover, early limbic activation followed by a late medial prefrontal component is entirely consistent with previous findings. ${ }^{9}$ The early limbic activations are thought to reflect memory retrieval and initial hedonic sensations and the delayed cortical activations are thought to reflect memory elaboration and reliving. ${ }^{9}$ Indeed, detailed visual imagery is a hallmark of vivid reliving, ${ }^{14}$ and visual cortical activations have been found to correlate positively with ratings of 'reliving' in previous studies.' Thus, the greater visual activations in the late recollection phase, when visual imagery becomes more pronounced, plus the reports of more visual and vivid recollections under psilocybin, support the hypothesis that psilocybin augments the neural processes underlying autobiographical recollection.

\section{Subjective experiences}

To give a sense of the qualitative difference between the recollective experiences in each condition, one participant commented after his second scan (placebo scan): 'I was focusing on my memories, but there wasn't the same kind of emotional engagement, and I didn't visualise them as realistically as I had done last week [under psilocybin].' When asked whether this meant that he was using his imagination more this time, he said:

'I think my imagination had a lot to do with it last time, but it seemed more vivid and real . . . I was more attached to the images and putting myself in the scenario than I was today. Today it was like ... I was thinking about it, it was nice, but it was nothing special - nothing new came out of it.

A number of participants also commented on the brevity of the recollection period and how memories only became more vivid towards the end of the $16 \mathrm{~s}$ under psilocybin. For example: 'I think the memories could develop more with more time - you could go deeper with more time'.

\section{Medial temporal lobe involvement in 'relivings'}

Despite these positive findings, there were some important negative findings in this study. None of our participants reported anything like the dramatic relivings that are described in the literature on psychedelics. ${ }^{2,12}$ We also did not find the hypothesised augmentation of medial temporal lobe responses under psilocybin - despite there being some compelling reasons to expect this: medial temporal lobe activations have been found during flashbacks in patients with $\mathrm{PTSD},{ }^{15}$ but the most compelling evidence for a medial temporal lobe involvement in relivings comes from invasive procedures. Wilder Penfield was the first to demonstrate that electrical stimulation of the temporal cortex could produce vivid recollections and reliving, ${ }^{16,17}$ and subsequent work has shown that the medial temporal lobes are especially sensitive to this effect. ${ }^{18}$ That medial temporal lobe stimulation can generate complex visual imagery is interesting and implies the occurrence of a spreading activation from the site of stimulation to posterior sensory regions. Evidence for this was found in a simultaneous stimulation-depth electroencephalography study, in which stimulation of the anterior medial temporal lobes produced complex visual imagery and a spreading activation to visual association regions in the theta frequency. ${ }^{19}$ Thus, our failure to find an exaggerated medial temporal lobe response under psilocybin may explain the absence of relivings, but it would be interesting to explore this issue further with connectivity analyses. For example, using dynamic causal modelling, ${ }^{20}$ we might predict an increased medial temporal lobe input to the visual cortex during recollection under psilocybin. We might also predict that connections from the medial prefrontal cortex to medial temporal lobe regions are decreased during recollection under psilocybin. Rostral anterior cingulate cortex blood flow was found to decrease during PTSD flashbacks (and to increase during dissociation), ${ }^{21}$ and in a previous psilocybin fMRI study by our group, we found an immediate, marked and sustained drop in medial prefrontal activity after intravenous psilocybin. ${ }^{5}$ The medial prefrontal cortex is known to exert a top-down inhibitory control over medial temporal lobe regions; ${ }^{6}$ thus, a decrease in medial prefrontal cortex activity may lead to a medial temporal lobe disinhibition. ${ }^{22}$

There are some likely explanations for why we did not observe the predicted relivings in this study. For example, in our efforts to minimise negative drug responses, we used only positive memory cues, but relivings are often linked to painful or conflict-laden memories. ${ }^{2}$ An improved design could use conflict-laden memory cues in patients in dynamic psychotherapy, so that any upsetting reactions could be worked through post-session. This would properly test the hypothesis that psychedelics facilitate emotional insight by lowering psychological defences. Also, increasing the duration of the recollection periods would allow a deeper level of introspection. However, despite these limitations, our decision to use only positive memory cues was not without reward; the primary hypothesis that psilocybin augments subjective and neural responses to positive autobiographical memory cues was supported and the experience was well tolerated by all of the participants. Indeed, consistent with previous studies, ${ }^{23}$ our sample reported significant improvements in subjective well-being after their psilocybin experience. Moreover, a positive correlation was found between ratings of subjective well-being and memory vividness. The following report from one participant testifies to this: 
'It being February and having endured the harsh months of a long British winter, I guess I'd been feeling a little blue lately, but right now I feel invigorated, recharged and inspired by the experience. . . What seems to be fuelling my optimism isn't just the drug experience, but the combination of it with refreshing my own memories; it's like I've been reminded of the affirmative aspects of my life.

\section{Implications for psychedelic-assisted psychotherapy}

The primary finding of this study was that psilocybin switched autobiographical memory activations in visual and other sensory regions from a pattern of deactivation to activation (Fig. 6). Participants also reported more vivid and visual recollections under psilocybin - which is consistent with the increased sensory activations. These effects may have implications for the use of psilocybin in psychotherapy. For example, psilocybin could be combined with positive memory cues as a treatment for depression - facilitating the recall of positive life events so to reverse pessimistic mind-sets. Support for such an idea comes from findings of decreased depression scores in patients with anxiety 6 months after a single psilocybin treatment ${ }^{24}$ and improvements in well-being and trait openness persisting in healthy volunteers up to 2 years after a single high dose of psilocybin. $^{23,25}$ Further supporting the case for psilocybin as a treatment option for depression, we recently observed marked decreases in medial prefrontal cortex activity after psilocybin. ${ }^{5}$ Hyperactivity in the medial prefrontal cortex in depression and its normalisation after effective treatment is a highly reliable finding. ${ }^{26}$

\section{Limitations}

The sample size in this study $(n=10)$ was small and may have encouraged false negatives. For example, we failed to find significant correlations between between-condition differences in brain activations and subjective ratings, which may have emerged in a larger sample. Another limitation was the self-selecting nature of the sample, which may have biased outcomes. Participants were required to have used psychedelics before, and many held prior assumptions about the positive value of these drugs. This may have contributed to the positive memory ratings, the reports of improved well-being post-administration, and the positive relationship between them (Fig. 6). Importantly, however, such biases cannot easily explain the increased brain activations to memories after psilocybin.

\section{Implications}

This study found increased subjective and neural responses to autobiographical memory cues under psilocybin. Greater activations were evident in visual and other sensory regions, which may explain why recollections were rated as more vivid and visual under psilocybin. Psychedelic drugs have a history of use in psychotherapy, linked to the hypothesis that they lower defences to facilitate access to salient emotions and memories. The results of this study provide initial support for this idea and a potential neurobiological mechanism is proposed: decreased medial prefrontal cortex activity leading to disinhibited limbic and sensory activity. We propose that psilocybin may be used in combination with cognitive strategies designed to reverse cognitive biases in depression - and we also suggest that it may be used in more classic dynamic therapy to assist the exploration and understanding of salient emotional themes.

\section{Funding}

This study received financial and intellectual support from the Beckley Foundation and financial support from the Neuropsychoanalysis Foundation, Multidisplinary Association for Psychedelic Studies (MAPS) and Heffter Research Institute.

\section{Acknowledgements}

We thank Eleanor Maguire, Karl Friston, Alison Diaper, Ann Rich, Sue Wilson, Andrea Malizia and David Jessop.

R. L. Carhart-Harris, PhD, Imperial College London, Neuropsychopharmacology Unit, and University of Bristol, Academic Unit of Psychiatry; R. Leech, PhD, Imperial College London; T. M. Williams, MD, University of Bristol, Academic Unit of Psychiatry; D. Erritzoe, MD, Imperial College London, Neuropsychopharmacology Unit; N. Abbasi, MD, University of Bristol, Academic Unit of Psychiatry; T. Bargiotas, MD, Oxford Health NHS Foundation Trust, Warneford Hospital, Oxford; P. Hobden, $\mathrm{PhD}$, Cardiff University Brain Research Imaging Centre (CUBRIC), School of

Psychology, Cardiff University; D. J. Sharp, MD, Imperial College London;

J. Evans, PhD, Cardiff University Brain Research Imaging Centre (CUBRIC), School of Psychology, Cardiff University; A. Feilding, The Beckley Foundation, Beckley Park Oxford; R. G. Wise, PhD, Cardiff University Brain Research Imaging Centre (CUBRIC), School of Psychology, Cardiff University; D. J. Nutt, MD, Imperial College London, Neuropsychopharmacology Unit, and University of Bristol, Academic Unit of Psychiatry, UK

Correspondence: R. L. Carhart-Harris, Imperial College London, Neuropsychopharmacology Unit, 5th Floor, Burlington Danes Building, $160 \mathrm{Du}$ Cane Road, London W12 0NN, UK. Email: r.carhart-harris@imperial.ac.uk

First received 19 Sep 2011, final revision 21 Nov 2011, accepted 30 Nov 2011

\section{References}

1 Cohen S. The Beyond Within - The LSD Story. Atheneum, 1964.

2 Sandison RA. A Century of Psychiatry, Psychotherapy and Group Analysis: A Search for Integration. Jessica Kingsley, 2001.

3 Halgren E, Walter RD, Cherlow DG, Crandall PH. Mental phenomena evoked by electrical stimulation of the human hippocampus formation and amygdala. Brain 1978; 101: 83-117.

4 Hagenaars MA, van Minnen A, Hoogduin KA. Reliving and disorganization in posttraumatic stress disorder and panic disorder memories. J Nerv Ment Dis 2009; 197: 627-30.

5 Carhart-Harris RL, Erritzoe D, Williams T, Stone JM, Read LJ, Colasanti A, et al. Neural correlates of the psychedelic state as determined by fMRI studies with psilocybin. Proc Natl Acad Sci USA 2012, in press (doi: 10.1073/ pnas.1119598109)

6 Milad MR, Quirk GJ. Neurons in medial prefrontal cortex signal memory for fear extinction. Nature 2002; 420: 70-4.

7 Greenberg DL, Rice HJ, Cooper JJ, Cabeza R, Rubin DC, LaBar KS. Co-activation of the amygdala, hippocampus and inferior frontal gyrus during autobiographical memory retrieval. Neuropsychologia 2005; 43 : 659-74.

8 Addis DR, Moscovitch M, Crawley AP, McAndrews MP. Recollective qualities modulate hippocampal activation during autobiographical memory retrieval. Hippocampus 2004; 14: 752-62.

9 Daselaar SM, Rice HJ, Greenberg DL, Cabeza R, LaBar KS, Rubin DC The spatiotemporal dynamics of autobiographical memory: neural correlates of recall, emotional intensity, and reliving. Cereb Cortex 2008; 18: $217-29$.

10 Carhart-Harris RL, Williams TM, Sessa B, Tyacke RJ, Rich AS, Feilding A, et al. The administration of psilocybin to healthy, hallucinogen-experienced volunteers in a mock-functional magnetic resonance imaging environment a preliminary investigation of tolerability. J Psychopharmacol 2011; 25: 1562-7.

11 Raichle ME, MacLeod AM, Snyder AZ, Powers WJ, Gusnard DA, Shulman GL. A default mode of brain function. Proc Natl Acad Sci USA 2001; 98: 676-82.

12 Grof S. Realms of the Human Unconscious. Souvenir Press, 1975.

13 de Araujo DB, Ribeiro S, Cecchi GA, Carvalho FM, Sanchez TA, Pinto JP, et al. Seeing with the eyes shut: neural basis of enhanced imagery following ayahuasca ingestion. Hum Brain Mapp 2011; Sep 16. Epub ahead of print (doi: 10.1002/hbm.21381)

14 Rubin DC, Schrauf RW, Greenberg DL. Belief and recollection of autobiographical memories. Mem Cognit 2003; 31: 887-901.

15 vermetten E, Schmahl C, Southwick SM, Bremner JD. Positron tomographic emission study of olfactory induced emotional recall in veterans with and without combat-related posttraumatic stress disorder. Psychopharmacol Bull 2007; 40: 8-30

16 Penfield W, Perot P. The brain's record of auditory and visual experience. A final summary and discussion. Brain 1963; 86: 595-696. 
17 Penfield W, Jasper S. Epilepsy and the Functional Anatomy of the Human Brain. Brown and Co., 1954.

18 Bancaud J, Brunet-Bourgin F, Chauvel P, Halgren E. Anatomical origin of déjà vu and vivid 'memories' in human temporal lobe epilepsy. Brain 1994; 117: 71-90.

19 Barbeau E, Wendling F, Régis J, Duncan R, Poncet M, Chauvel P, et al. Recollection of vivid memories after perirhinal region stimulations: synchronization in the theta range of spatially distributed brain areas. Neuropsychologia 2005; 43: 1329-37.

20 Friston KJ, Harrison L, Penny W. Dynamic causal modelling. Neurolmage 2003; 19: 1273-302.

21 Hopper JW, Frewen PA, van der Kolk BA, Lanius RA. Neural correlates of reexperiencing, avoidance, and dissociation in PTSD: symptom dimensions and emotion dysregulation in responses to script-driven trauma imagery. J Trauma Stress 2007; 20: 713-25.
22 Carhart-Harris RL, Friston KJ. The default-mode, ego-functions and freeenergy: a neurobiological account of Freudian ideas. Brain 2010; 133: 1265-83.

23 Griffiths R, Richards W, Johnson M, McCann U, Jesse R. Mystical-type experiences occasioned by psilocybin mediate the attribution of personal meaning and spiritual significance 14 months later. J Psychopharmacol 2008; 22: 621-32.

24 Grob CS, Danforth AL, Chopra GS, Hagerty M, McKay CR, Halberstadt AL, et al. Pilot study of psilocybin treatment for anxiety in patients with advanced-stage cancer. Arch Gen Psychiatry 2011; 68: 71-8.

25 MacLean KA, Johnson MW, Griffiths RR. Mystical experiences occasioned by the hallucinogen psilocybin lead to increases in the personality domain of openness. J Psychopharmacol 2011; 25: 1143-61.

26 Hamani C, Mayberg H, Stone S, Laxton A, Haber S, Lozano AM. The subcallosal cingulate gyrus in the context of major depression. Biol Psychiatry 2011; 69: 301-8.

Two British colonial administrators, Sir Frank Swettenham and Sir Hugh Clifford, wrote about amok in 19th-century Malaya from a sociological perspective. Clifford noted that 'amok is a result of a condition of the mind which is described in the vernacular as sakit hati - sickness of the liver, the centre of sensitiveness'. He felt it was caused by an emotional problem which precipitated the sakit hati or Malay equivalent of depression, leading to the homicidal-suicidal rage. The fate of the person was discussed by swettenham: 'he is regarded as a dangerous beast. As a rule he is not taken alive'. 